\title{
SUPERFICIE DE SUELOS POTENCIALMENTE DISPONIBLE PARA FORESTACIÓN REGIÓN DEL BIOBÍO
}

\author{
Ávila, Alberto ${ }^{1}$ y Muñoz, Juan Carlos ${ }^{1}$
}

\section{RESUMEN}

El objetivo de este estudio es determinar la superficie de suelos de aptitud preferentemente forestal potencialmente disponible para forestación y su ubicación en la región del Biobío.

Mediante un Sistema de Información Geográfico (SIG) se procesó coberturas digitales provenientes de distintas fuentes de información, correspondientes a plantaciones forestales, bosque nativo y capacidad de uso de los suelos.

Como resultado se obtuvo las superficies potencialmente disponibles para la forestación en la región, según provincias y comunas, índice de desarrollo humano (IDH), clase capacidad de uso del suelo y categorías de erosión. 351.540 ha.

La superficie de suelos potencialmente disponible para forestación en la región es de

Palabras clave: Plantaciones forestales, Región del Biobío, Superficie disponible para forestación, IDH.

\section{SUMMARY}

Main objective of this study is to determine the forest soil areas potentially available to afforestation and its location at the Biobío region.

Through a Geographic Information System (GIS) different digital covers, including planted forests, native forests and soil use capacity class were processed.

The obtained results are the forest soil potential areas available to afforestation in the region according to provinces and communes, human development index (HDI), soil use capacity and erosion categories.

The total soil area potentially available to afforestation in the region is 351,540 ha.

Key words: Planted forests. Biobío Region, Available area to afforestation, HDI.

\footnotetext{
${ }^{1}$ Ingenieros forestales, investigadores, Instituto Forestal, Sede Biobío. Chile. aavila@infor.cl jmunoz@infor.cl
} 


\section{INTRODUCCIÓN}

El sector forestal chileno se puede definir como la integración de los recursos forestales suelos y bosques nativos y plantados con todas sus funciones ambientales, económicas y sociales; las grandes, medianas y pequeñas industrias asociadas a estos recursos; los propietarios; las instituciones del Estado; y todo el recurso humano directa e indirectamente involucrado en el bosque, la industria y la instituciones; integración que constituye un importante pilar del desarrollo nacional.

El $45 \%$ de la superficie continental de Chile corresponde a suelos cuyo uso potencial es forestal $\left(33,8 \mathrm{MM}^{2} \mathrm{ha}\right)$, el $21 \%$ del territorio está cubierto por bosques nativos (13,5 $\mathrm{MM}$ ha) y plantaciones (2,4 MM ha), lo que conforma una cubierta forestal de unos 16 millones de hectáreas (INFOR, 2014).

El Estado mantiene bajo conservación o protección una superficie de 14,5 MM ha dentro del Sistema Nacional de Áreas Silvestres Protegidas (19\% de la superficie nacional), en el cual están representados los principales ecosistemas forestales del país, incluyendo unos $4 \mathrm{MM}$ ha de bosques nativos.

Actualmente no existe deforestación, la sustitución de bosques nativos no está permitida, toda intervención sobre los bosques está regulada por el Estado y la reforestación es obligatoria.

Los bosques plantados, que representan el $15 \%$ de la cubierta forestal nacional, son hoy la base de una desarrollada y creciente industria forestal, que consume unos $40 \mathrm{MM} \mathrm{m}^{3}$ /año, casi en su totalidad proveniente de las plantaciones, para la producción de pulpa, madera aserrada, tableros y otros productos.

El sector forestal genera anualmente unos US\$ 6 mil millones como retorno de exportaciones y da empleo directo a más de 120 mil personas e indirecto a más 180 mil personas (INFOR, 2014; CORMA, 2014; Lignum, 2014).

Sin embargo, desde los tiempos de La Colonia y hasta mediados del siglo pasado, la apertura de tierras para uso agrícola y ganadero, grandes incendios forestales y la sobre utilización de los bosques nativos dejaron como herencia extensas superficies de suelos forestales desarbolados y en procesos de erosión y bosques nativos degradados, fraccionados y empobrecidos en su composición de especies, situación que dejó a la forestación de los primeros y a la recuperación y manejo sostenible de los segundos como grandes desafíos sectoriales.

Estos desafíos fueron ya abordados en la segunda mitad del siglo pasado, a través del establecimiento de plantaciones y el desarrollo de la capacidad industrial derivada con grandes esfuerzo públicos y privados, la creación de una institucionalidad forestal pública y la regulación estatal sobre el uso de los bosques, sin embargo se mantienen estos desafíos como las principales metas sectoriales para el presente siglo.

En lo referente a las plantaciones forestales, la investigación, la legislación de fomento a la forestación y al manejo de las nuevas plantaciones y los esfuerzos del sector público al principio y principalmente del sector privado después han permitido la creación de nuevos recursos forestales mediante bosques plantados a un ritmo medio cercano a las $100 \mathrm{Mha} / \mathrm{año}$ desde mitad de los años 70 del siglo pasado.

Esta tasa anual de plantación tenía inicialmente una fuerte componente de forestación, pero a medida que el consumo industrial aumenta la componente reforestación también lo hace y la tasa de incremento de la superficie plantada es menor.

\footnotetext{
${ }^{2}$ MM: Millones M: Miles
} 
Durante los últimos años la legislación de fomento a la forestación ha sufrido interrupciones, su vigencia concluía en 1994, el cuerpo legal (DL $N^{\circ} 701$ de 1974) fue modificado y extendido hasta que finalmente caduca en 2012.

Actualmente se encuentra en trámite legislativo una nueva extensión de esta legislación de fomento y se planea al corto plazo el estudio, elaboración y promulgación de una nueva legislación al respecto.

Estas interrupciones en la aplicación de la política de fomento a las plantaciones ha reducido aún más la forestación y si bien la tasa de plantación se mantiene alta (95 Mha en 2013) la componente forestación cae sustancialmente (6.600 ha en 2013) (INFOR, 2014) y esto afecta principalmente al segmento de pequeños y medianos propietarios (PYMP), quienes habían tenido una interesante participación, reuniendo casi un tercio de la superficie plantada en el país.

Respecto de la superficie disponible para forestación en el país no existen cifras debidamente fundadas, aunque la cifra de uso potencial forestal de los suelos (33,8 MM ha), comparada con la actual cubierta forestal del país (16 MM ha), sugiere que la superficie disponible para nuevos bosques plantados es muy importante y con frecuencia son mencionadas cifras de varios millones de hectáreas.

No obstante, las grandes superficies consideradas como potencialmente disponibles se reducen en la práctica en forma importante por diversas razones entre las que se encuentran ubicación, altitud, accesibilidad, limitantes climáticas para las principales especies forestales actualmente en uso, régimen de propiedad y otras.

Contrariamente, estas superficies se pueden incrementar en alguna medida por suelos agrícolas o ganaderos de secano, en los cuales estas actividades ya no rentan debido a la degradación de los suelos, su ubicación u otras razones.

El Estado ha desplegado importantes esfuerzos para mantener una fuerte base de información en materia de caracterización, localización y cuantificación de los recursos forestales del país.

INFOR en los años 60 del siglo pasado desarrolla la tipología del bosque nativo, definiendo y caracterizando los Tipos Forestales, y realiza los primeros inventarios forestales regionales y nacionales que localizan y dimensionan estos recursos.

Posteriormente, INFOR ha mantenido un programa de investigación permanente en torno al Monitoreo de los Ecosistemas Forestales conformados tanto por los bosque nativos como los plantados, mediante el cual actualiza la superficie de plantaciones anualmente y determina sus existencias madereras en períodos de 3 a 4 años; y trabajo similar desarrolla respecto de los boques nativos actualizando sus existencias volumétricas en períodos también de 3 a 4 años.

Por su parte, el Proyecto CONAF-CONAMA-BIRF (1999) desarrolla el Catastro y Evaluación de Recursos Vegetacionales Nativos de Chile definiendo localización y superficie de estos recursos.

En consecuencia, el país dispone de información completa y actualizada sobre sus recursos forestales, su ubicación, sus características y sus existencias, información desagregada a nivel regional, provincial y comunal con la cartografía digital correspondiente. Sin embargo, no se han desarrollado estudios respecto de la superficie potencialmente disponible para ampliar las plantaciones forestales en el país.

INFOR aborda en 2014 el trabajo de determinación de la superficie potencialmente disponible para forestación en el país, empezando por la región del Biobío, que tiene ya más de 0,9 
millones de hectáreas plantadas y reúne gran parte de la capacidad industrial instalada en el país para el aprovechamiento de estos recursos.

El estudio fue llevado a cabo en la sede Biobío de INFOR en Concepción dentro del programa institucional Inventario Forestal Continuo de las plantaciones.

Se empleó para ello diferentes coberturas digitales y un sistema de información geográfica con el objeto de delimitar aquellas superficies de suelos forestales actualmente desprovistos de plantaciones o bosques nativos por provincias y comunas.

Las superficies así segregadas fueron caracterizadas según capacidad de uso de los suelos y categorías de erosión de estos y complementariamente se incorporó una variable de carácter social dada por un índice de desarrollo humano. Los resultados obtenidos son los que se entregan en este artículo.

Pasos siguientes en esta línea de trabajo sería repetirlo en otras regiones hasta completar en primera instancia aquellas comprendidas entre Valparaíso y Los Lagos, y en una segunda instancia incorporar Aysén y Magallanes, y tal vez también Coquimbo.

Progresivamente se podrán incorporar otras variables relacionadas con suelo, clima, tenencia de la tierra, y otras con el fin de extraer de la superficie potencialmente disponible aquella realmente disponible y, más aún, definir por ejemplo áreas prioritarias de forestación, especies recomendables y otros elementos de juicio que puedan guiar la ampliación de las plantaciones forestales en el país según objetivos específicos en cada caso, sean estos productivos o ambientales.

\section{OBJETIVOS}

El objetivo general del estudio es determinar la superficie de suelos forestales potencialmente disponible para forestación y su distribución en la región del Biobío.

Más específicamente, se intenta determinar la superficie de suelos potencialmente disponible para forestación según clase de capacidad de uso y categoría de erosión de estos, división administrativa de la región (provincias y comunas) y su vinculación con una dimensión social determinada por el índice de desarrollo humano (IDH).

\section{MATERIAL Y MÉTODO}

Fueron empleadas diferentes coberturas digitales, de distintas fuentes, que fueron procesadas utilizando el programa ArcGis ${ }^{\mathrm{TM}}$ y proyectadas al Datum WGS84 huso 18.

Las coberturas digitales empleadas son las siguientes:

-Clase Capacidad de Uso de los Suelos y Categorías de Erosión (CIREN, 1999)

-Plantaciones forestales a diciembre del año 2012 (INFOR, 2013)

-Catastro de Bosque Nativo VIII región, Actualización (CONAF, 2008)

-Limites Comunales región del Biobío (ODEPA, 2013)

Las coberturas de suelos, bosque nativo y límites comunales fueron obtenidas del Geoportal Infraestructura de Datos Espaciales (IDE) del Ministerio de Agricultura, en donde están 
las coberturas de CIREN 1999, la de CONAF 2008 y la de ODEPA 2013.

Para incorporar la componente social a este estudio se utilizó la información disponible sobre el Índice de Desarrollo Humano (IDH) al año 2003 para las comunas de Chile, elaborada por el Programa de las Naciones Unidas para el Desarrollo (PNUD) en conjunto con el Ministerio de Planificación (MIDEPLAN).

Se entiende por desarrollo humano al proceso mediante el cual se aumentan las capacidades y opciones de las personas.

El IDH se concentra en medir las capacidades humanas en tres dimensiones es enciales; salud, educación e ingresos. Sus valores oscilan entre 0 y 1 y este último valor representa la situación ideal (PNUD-MIDEPLAN, 2005).

La característica principal de esta clasificación es su ordenamiento relativo, es decir, se ordenaron las comunas respecto de su valor de IDH, y luego se formaron cinco grupos con igual número de comunas cada uno (quintiles).

El primer grupo corresponde al $20 \%$ de las comunas con menor valor de IDH, el segundo al $20 \%$ de comunas que le sigue según los valores de desarrollo humano, y así sucesivamente hasta el grupo 5 , que corresponde al $20 \%$ de las comunas con mayor valor de IDH.

Cada grupo se identificó con los conceptos muy bajo, bajo, medio, alto y muy alto nivel de logro (PNUD-MIDEPLAN, 2005).

La Figura $N^{\circ} 1$ muestra los resultados de este estudio para la región del Biobío.

Es preciso señalar que con posterioridad al año 2003, año del estudio de IDH, fueron creadas las comunas de Hualpén y Alto Biobío.

En los casos de estas nuevas comunas se asumió el valor IDH de la comuna de la cual provenían, Talcahuano y Santa Bárbara, respectivamente.

La cobertura digital de plantaciones forestales corresponde a la generada dentro del programa Inventario Forestal Continuo de INFOR, en su parte actualización de plantaciones (2012), y se refiere a la superficie de plantaciones en pie al momento de la actualización.

Esta última cobertura incluye la información proveniente de las empresas en convenio con INFOR y aquella otra sobre pequeños y medianos propietarios (PYMP) que INFOR levanta anualmente.

La cobertura de suelos de CIREN, indica las Clases de Capacidad de Uso de estos, I a VIII, definidas como:

I: $\quad$ Suelos agrícolas con pocas limitaciones.

II: $\quad$ Suelos con ligeras limitaciones al desarrollo de cultivos (pendientes hasta $5 \%$ )

III: Suelos suavemente inclinados (pendientes hasta 8\%)

IV: $\quad$ Suelos que pueden presentar riesgo de erosión por pendientes (hasta 15\%) o profundidad no superior a $40 \mathrm{~cm}$. Es la última categoría de suelos arables.

V: $\quad$ Suelos inundados pobremente drenados a muy pobremente 
drenados.

VI: Suelos cuyo uso es ganadero o forestal y son moderadamente escarpados

VII: $\quad$ Suelos de uso fundamentalmente forestal o ganadero y son escarpados.

VIII: $\quad$ Suelos de uso limitado a vida silvestre, recreación o protección de hoyas hidrográficas.

NC: $\quad$ Categoría adicional "No Corresponde", que se refiere a situaciones en que a los suelos no se les asigna algún uso productivo, como zonas urbanas, lagunas, cajas de río.

Esta cobertura no posee información en algunos sectores de la Cordillera de Los Andes, por lo que no es posible estimar la superficie potencial en dichas áreas.

Las categorías de erosión, también incorporadas en la cobertura de suelos de CIREN, son las siguientes:

Sin erosión:

Erosión ligera:

Erosión moderada:

Erosión severa:

Erosión muy severa:
Suelos que no presentan alteraciones o signos de pérdidas de suelo.

Suelos poco alterados, ligeramente inclinados $u$ ondulados, que pueden tener en torno al $20 \%$ de pérdida de suelo.

Erosión laminar o de manto de nivel medio. El suelo original se ha perdido entre 40 y $60 \%$.

Erosión laminar o de manto intensiva. La pérdida de suelo original es de 60 a $80 \%$ y presenta ocasionalmente surcos o cárcavas.

Zonas donde se visualiza el subsuelo o el material parental. La pérdida de suelo original es sobre el $80 \%$ y normalmente se encuentran surcos y cárcavas activas.

Las descripciones de las categorías de erosión fueron tomadas de CIREN (2006) y de Flores et al. (2010).

De los campos de Clase de Capacidad de Uso que entrega la cobertura digital de suelos se seleccionó aquellos considerados como No Arables, es decir aquellos que no son aptos para el laboreo y cuyo uso recomendado es preferentemente el forestal.

En la región estos suelos no arables suman 2.097 .030 ha y corresponden a 334.095 ha clase VI, 1.290.217 ha clase VII y 472.718 ha clase VIII (Figura $\mathrm{N}^{\circ} 2$ ): 


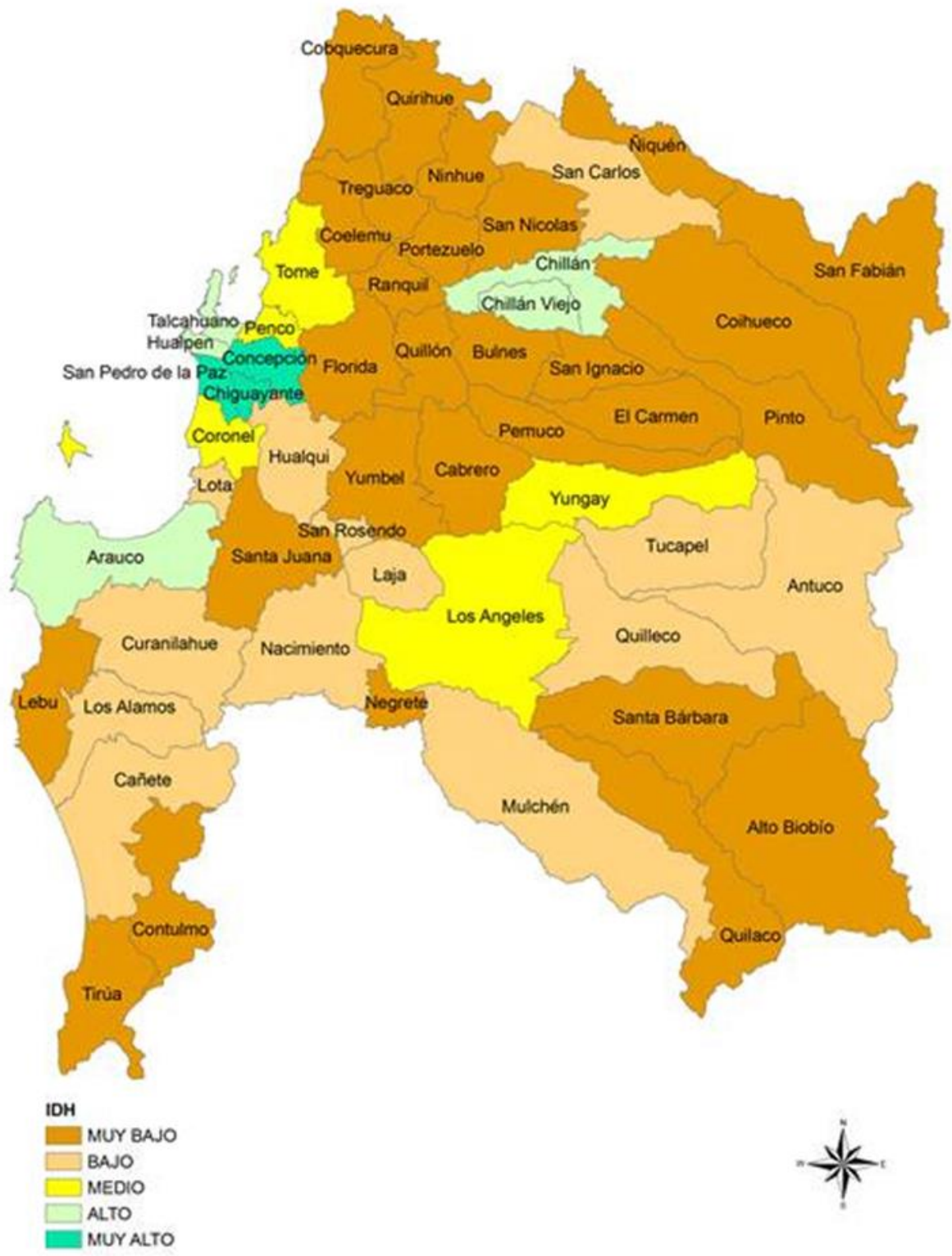

Figura 1

ÍNDICE DE DESARROLLO HUMANO POR COMUNAS, REGIÓN DEL BIOBíO 


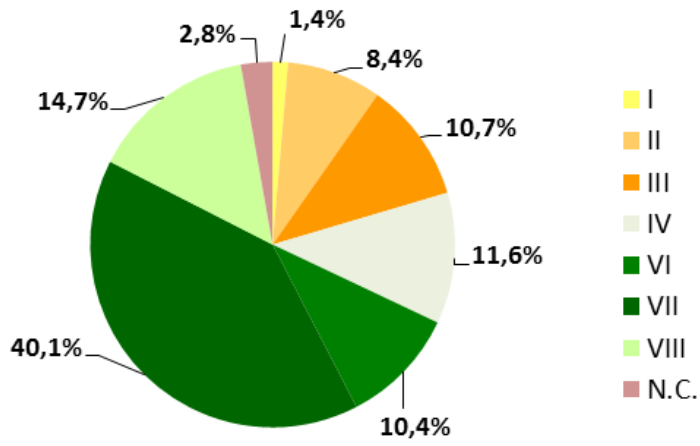

Figura $\mathrm{N}^{\circ} 2$

DISTRIBUCIÓN PORCENTUAL DE SUPERFICIE POR CLASE DE CAPACIDAD USO DE SUELO

A la superficie segregada con la restricción Clase de Capacidad de Uso del Suelo, se le descontó también la Clase VIII, dado que corresponde a suelos cuyo uso está reducido a la protección y vida silvestre.

De igual forma se quitaron los polígonos correspondientes a las siguientes variaciones especiales de suelo: Corrientes de lava, terrenos rocosos, pantanos (humedales), ríos, quebradas, esteros y escarpes, obteniéndose de esta forma la cobertura de suelos con capacidad de uso forestal de la región del Biobío.

A la superficie así obtenida se le descontó la cobertura de plantaciones forestales, incluyendo tanto los polígonos correspondientes a plantaciones en pie y también aquellos de cosechas forestales, dado que de acuerdo la legislación vigente deben ser reforestados en un plazo de 3 años desde la corta.

Esto último se realizó mediante el comando de análisis ArcGis ${ }^{\mathrm{TM}}$ Erase, que sustrae la superficie en donde se sobreponen ambas coberturas digitales y crea una nueva cobertura.

Similar procedimiento se empleó para descontar la cubierta dada por el catastro de bosque nativo, restando los polígonos de uso actual bosque nativo, y se extrajeron igualmente las superficies de áreas silvestres protegidas del Estado (SNASPE), nieves y glaciares, áreas urbanas e industriales. Finalmente se descontaron también aquellos polígonos producidos durante el proceso cuya superficie era inferior a 1 ha.

La cobertura final resultante corresponde a la superficie potencialmente disponible para la forestación, la cual mediante el proceso denominado Intersect en ArcGis ${ }^{\mathrm{TM}}$ se sobrepuso con la cobertura de límites comunales, la que a su vez está vinculada a una categoría de IDH.

\section{RESULTADOS}

Al aplicar la primeras restricciones, suelos arables y clase VIII, se obtiene la cobertura de suelos de aptitud forestal de la región y entrega como resultado una superficie total de 1.456 .866 ha. Se incorporaron después las coberturas de capacidad de uso, categorías de erosión y división administrativa según provincias, y la superficie así obtenida corresponde a suelos de Clases de 
Capacidad de Uso VI y VII, muy mayoritariamente Clase VII que son los suelos típicamente forestales $(77 \%)$ y representan aproximadamente el $40 \%$ del área total regional, y sin cubierta forestal por provincias y comunas (Cuadro $\mathrm{N}^{\circ} 1$ y Figura $\mathrm{N}^{\circ} 4$ ).

Cuadro $\mathrm{N}^{\circ} 1$

SUPERFICIE DE SUELOS DE APTITUD FORESTAL, SEGÚN CLASE DE CAPACIDAD DE USO Y CATEGORÍA DE EROSIÓN POR PROVINCIA

\begin{tabular}{|c|c|c|c|c|c|c|}
\hline \multirow{3}{*}{$\begin{array}{c}\text { Clase } \\
\text { Capacidad } \\
\text { Uso del } \\
\text { Suelo }\end{array}$} & \multirow{3}{*}{$\begin{array}{c}\text { Categoría } \\
\text { Erosión }\end{array}$} & \multicolumn{4}{|c|}{ Provincia } & \multirow{2}{*}{ Total } \\
\hline & & Arauco & Biobío & Concepción & Ñuble & \\
\hline & & \multicolumn{5}{|c|}{ (ha) } \\
\hline \multirow{3}{*}{ VI } & Ligera & 46.551 & 18.479 & 26.979 & 39.552 & 131.561 \\
\hline & Moderada & 0 & 54.440 & 3.282 & 37.455 & 95.177 \\
\hline & Sin Erosión & 14.031 & 61.562 & 301 & 31.463 & 107.357 \\
\hline \multicolumn{2}{|l|}{ Total VI } & 60.582 & 134.481 & 30.562 & 108.470 & 334.095 \\
\hline \multirow{6}{*}{ VII } & Ligera & 248.163 & 20.621 & 53.875 & 49.953 & 372.612 \\
\hline & Moderada & 55.119 & 81.676 & 161.369 & 114.129 & 412.293 \\
\hline & Severa & 2.166 & 55.479 & 30.211 & 90.842 & 178.698 \\
\hline & Muy Severa & 0 & 29.237 & 10.892 & 30.237 & 70.366 \\
\hline & Sin Erosión & 8.061 & 25.226 & 2.119 & 7.078 & 42.484 \\
\hline & S/Información & 16.991 & 28.304 & 1.023 & 0 & 46.318 \\
\hline \multicolumn{2}{|l|}{ Total VII } & 330.500 & 240.543 & 259.489 & 292.239 & 1.122 .771 \\
\hline \multicolumn{2}{|l|}{ Total } & 391.082 & 375.024 & 290.051 & 400.709 & 1.456 .866 \\
\hline
\end{tabular}

En el procesamiento se incorporaron las cubiertas de plantaciones forestales (en pie y cosechadas) y la correspondiente a bosques nativos, descontándose estas superficies a la de suelos de aptitud forestal, para obtener así la superficie potencialmente disponible para forestación en la región, que arroja un total de 351.540 ha.

Esta superficie potencialmente disponible para forestación está constituida en un 70,8\% por suelos de Clase de Capacidad de Uso VII y un 29,2\% por suelos de Clase VI (Cuadro $N^{\circ} 2$ y Figuras $\mathrm{N}^{\circ} 4$ y $\mathrm{N}^{\circ} 5$ ). 


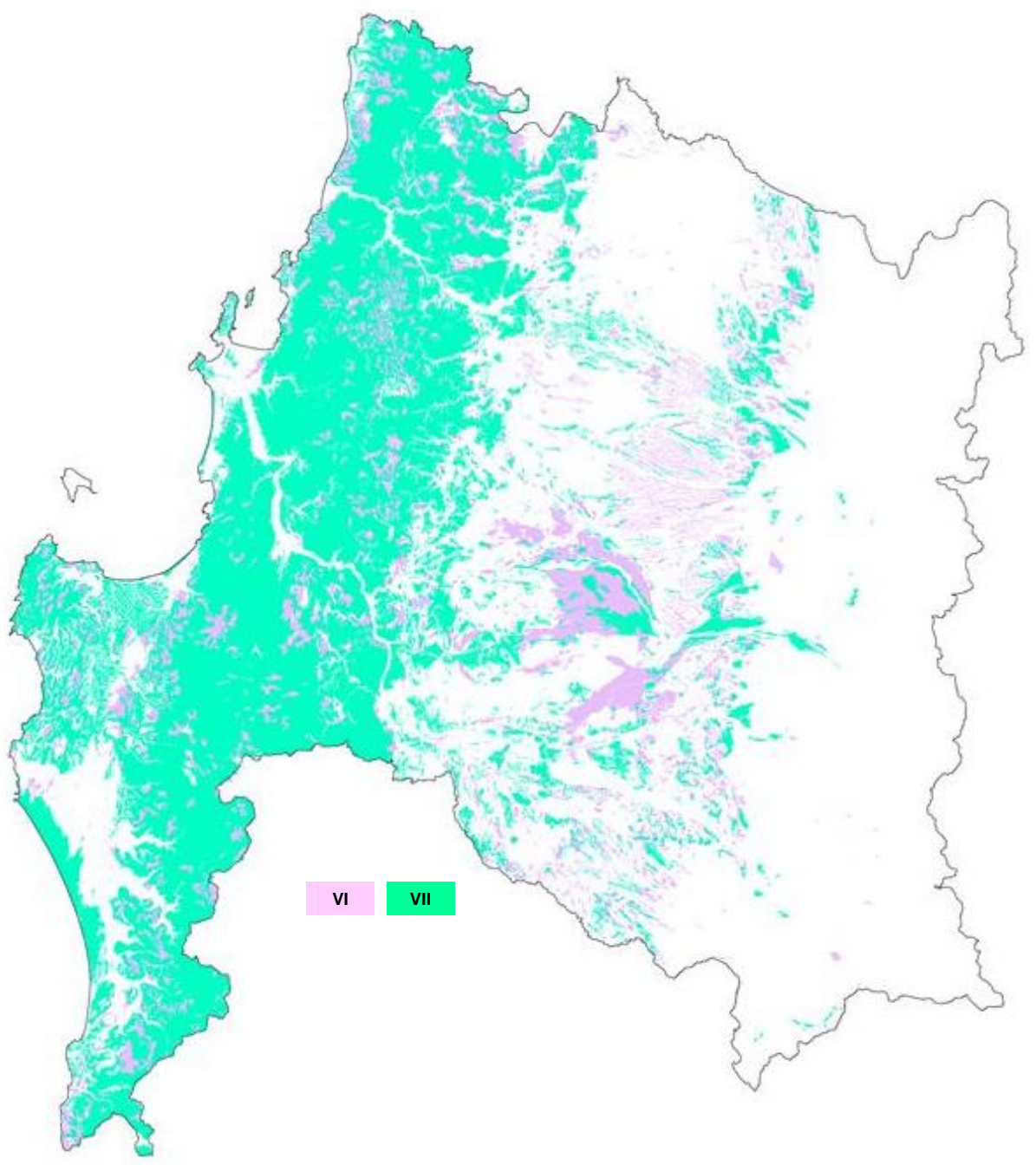

Figura $\mathrm{N}^{\circ} 4$

SUELOS DE APTITUD FORESTAL REGIÓN DEL BIOBÍO 
SUPERFICIE DE SUELOS POTENCIALMENTE DISPONIBLES PARA FORESTACIÓN SEGÚN CLASE DE CAPACIDAD DE USO Y CATEGORÍA DE EROSIÓN POR PROVINCIA

\begin{tabular}{|c|c|c|c|c|c|c|}
\hline \multirow{3}{*}{$\begin{array}{c}\text { Capacidad } \\
\text { Uso del } \\
\text { Suelo }\end{array}$} & \multirow{3}{*}{$\begin{array}{l}\text { Categoría } \\
\text { Erosión }\end{array}$} & \multicolumn{4}{|c|}{ Provincia } & \multirow{2}{*}{ Total } \\
\hline & & Arauco & Biobío & Concepción & Ñuble & \\
\hline & & \multicolumn{5}{|c|}{ (ha) } \\
\hline \multirow{3}{*}{ VI } & Ligera & 5.222 & 8.995 & 7.797 & 19.858 & 41.872 \\
\hline & Moderada & 0 & 14.299 & 2.469 & 12.949 & 29.717 \\
\hline & Sin Erosión & 6.178 & 14.259 & 198 & 10.334 & 30.969 \\
\hline \multicolumn{2}{|l|}{ Total VI } & 11.400 & 37.553 & 10.464 & 43.141 & 102.558 \\
\hline \multirow{6}{*}{ VII } & Ligera & 29.375 & 3.093 & 3.067 & 8.976 & 44.511 \\
\hline & Moderada & 3.160 & 16.212 & 30.226 & 23.699 & 73.297 \\
\hline & Severa & 602 & 17.666 & 8.857 & 42.714 & 69.839 \\
\hline & Muy Severa & 0 & 13.904 & 5.929 & 11.844 & 31.677 \\
\hline & Sin Erosión & 4.326 & 7.580 & 986 & 3.940 & 16.832 \\
\hline & S/Información & 3.637 & 8.821 & 368 & 0 & 12.826 \\
\hline \multicolumn{2}{|l|}{ Total VII } & 41.100 & 67.276 & 49.433 & 91.173 & 248.982 \\
\hline \multicolumn{2}{|l|}{ Total } & 52.500 & 104.829 & 59.897 & 134.314 & 351.540 \\
\hline
\end{tabular}

De acuerdo a la división administrativa por provincias, es posible apreciar que la mayor superficie se encuentra en la provincia de Ñuble $(38,2 \%)$ y la siguen la provincia de Biobío $(29,8 \%)$, la provincia de Concepción (17,1\%) y la provincia de Arauco (14,9\%).

Respecto de las categorías de erosión, en los suelos clase VI el 69,8\% de la superficie presenta erosión ligera a moderada, en tanto que en aquellos de clase VII la erosión es severa a muy severa en un $40,8 \%$ de su superficie.

El deterioro de los suelos resalta en las provincias de Nuble y Biobío, en donde alrededor de la mitad $(59,6 \%$ y $46,9 \%$, respectivamente) de la superficie de suelos clase VII potencialmente disponibles para forestación muestra erosión severa a muy severa.

La provincia de Arauco en tanto muestra la menor degradación de suelos, con solo el $1,4 \%$ de la superficie de suelos clase VII potencialmente disponibles para forestación con erosión severa.

Se incorporó finalmente la división administrativa de la región por comunas y se obtuvo la distribución de superficies potencialmente disponibles para forestación según comunas, clases de capacidad de uso de los suelos, categorías de erosión y sus correspondientes categorías de índice de desarrollo humano (IDH), para cada una de las provincias de la región del Biobío (Cuadros $\mathrm{N}^{\circ} 3$, $\mathrm{N}^{\circ} 4, \mathrm{~N}^{\circ} 5$ y $\mathrm{N}^{\circ} 6$ ). 


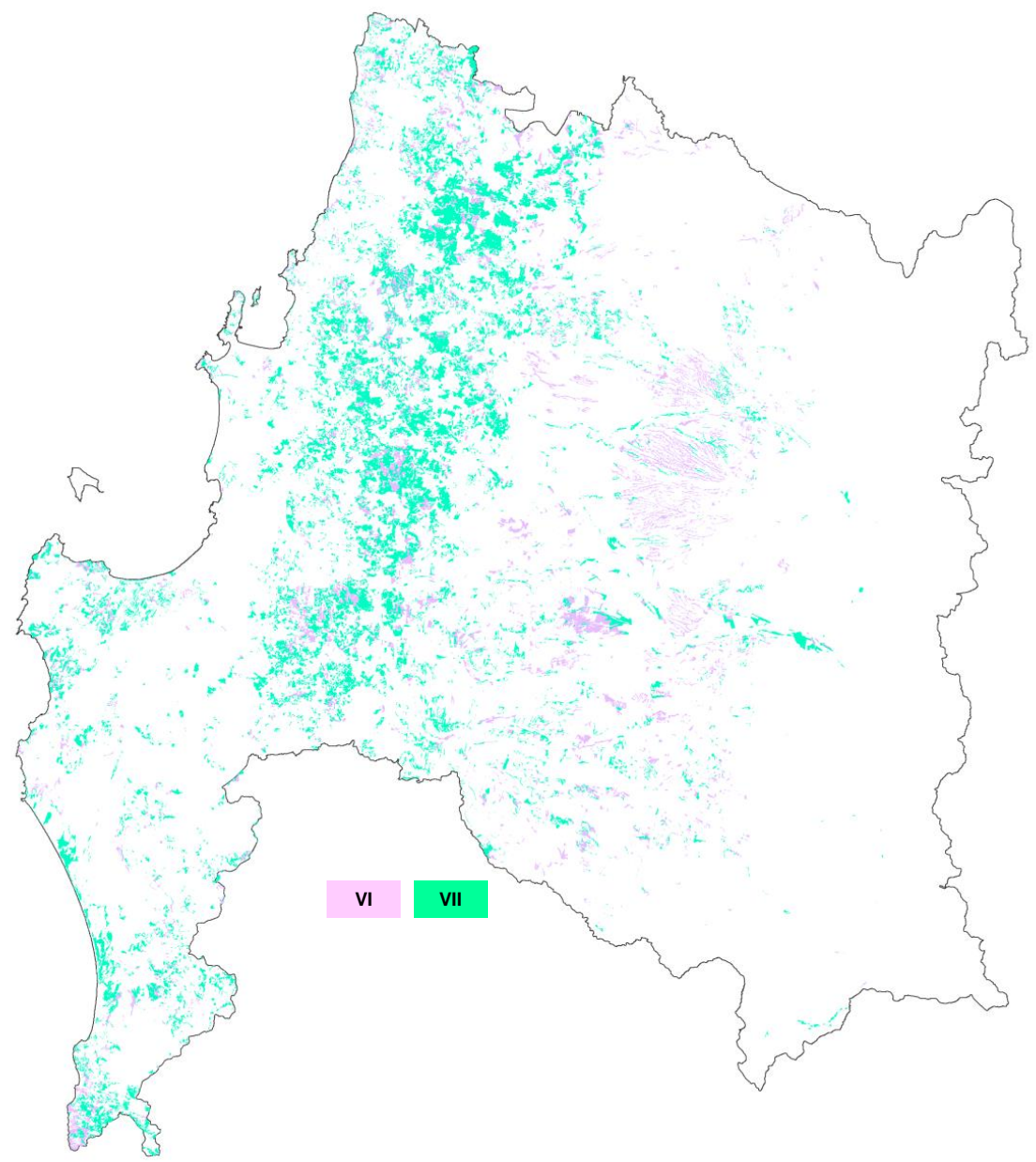

Figura $\mathrm{N}^{\circ} 5$

SUPERFICIE POTENCIALMENTE DISPONIBLE PARA FORESTACIÓN

POR CLASE DE CAPACIDAD USO DEL SUELO 
Cuadro $\mathrm{N}^{\circ} 3$

SUPERFICIE POTENCIALMENTE DISPONIBLE PARA FORESTACIÓN SEGÚN COMUNA, IDH, CLASES DE CAPACIDAD DE USO DE LOS SUELOS Y CATEGORÍAS DE EROSIÓN

PROVINCIA DE ARAUCO

\begin{tabular}{|c|c|c|c|c|c|c|c|c|}
\hline \multirow{3}{*}{ Comuna } & \multirow{3}{*}{ IDH } & \multirow{3}{*}{$\begin{array}{c}\text { Capacidad } \\
\text { Uso del } \\
\text { Suelo }\end{array}$} & \multicolumn{5}{|c|}{ Categorías de Erosión } & \multirow{2}{*}{ Total } \\
\hline & & & $\mathbf{L}$ & M & $\mathbf{S}$ & SE & SI & \\
\hline & & & \multicolumn{6}{|c|}{ (ha) } \\
\hline \multirow{2}{*}{ Arauco } & \multirow{2}{*}{ Alto } & VI & 1.094 & 0 & 0 & 1.653 & 0 & 2.747 \\
\hline & & VII & 8.460 & 875 & 0 & 133 & 104 & 9.573 \\
\hline \multicolumn{3}{|l|}{ Total Arauco } & 9.554 & 875 & 0 & 1.786 & 104 & 12.320 \\
\hline \multirow{2}{*}{ Cañete } & \multirow{2}{*}{ Bajo } & $\mathrm{VI}$ & 587 & 0 & 0 & 378 & 0 & 965 \\
\hline & & VII & 2.996 & 209 & 0 & 2.058 & 1.427 & 6.689 \\
\hline \multicolumn{3}{|l|}{ Total Cañete } & 3.583 & 209 & 0 & 2.435 & 1.427 & 7.655 \\
\hline \multirow{2}{*}{ Contulmo } & \multirow{2}{*}{ Muy Bajo } & $\mathrm{VI}$ & 1.473 & 0 & 0 & 222 & 0 & 1.695 \\
\hline & & VII & 6.339 & 11 & 0 & 0 & 0 & 6.351 \\
\hline \multicolumn{3}{|c|}{ Total Contulmo } & 7.812 & 11 & 0 & 222 & 0 & 8.045 \\
\hline \multirow{2}{*}{ Curanilahue } & \multirow{2}{*}{ Bajo } & $\mathrm{VI}$ & 475 & 0 & 0 & 0 & 0 & 475 \\
\hline & & VII & 915 & 1.524 & 602 & 0 & 0 & 3.041 \\
\hline \multicolumn{3}{|c|}{ Total Curanilahue } & 1.390 & 1.524 & 602 & 0 & 0 & 3.516 \\
\hline \multirow{2}{*}{ Lebu } & \multirow{2}{*}{ Muy Bajo } & $\mathrm{VI}$ & 176 & 0 & 0 & 1.272 & 0 & 1.449 \\
\hline & & VII & 2.446 & 391 & 0 & 76 & 727 & 3.640 \\
\hline \multicolumn{3}{|l|}{ Total Lebu } & 2.622 & 391 & 0 & 1.348 & 727 & 5.089 \\
\hline \multirow{2}{*}{ Los Álamos } & \multirow{2}{*}{ Bajo } & $\mathrm{VI}$ & 239 & 0 & 0 & 60 & 0 & 299 \\
\hline & & VII & 1.169 & 149 & 0 & 118 & 1.379 & 2.815 \\
\hline \multicolumn{3}{|c|}{ Total Los Álamos } & 1.408 & 149 & 0 & 178 & 1.379 & 3.113 \\
\hline \multirow{2}{*}{ Tirúa } & \multirow{2}{*}{ Muy Bajo } & $\mathrm{VI}$ & 1.178 & 0 & 0 & 2.593 & 0 & 3.771 \\
\hline & & VII & 7.050 & 0 & 0 & 1.941 & 0 & 8.991 \\
\hline \multicolumn{3}{|l|}{ Total Tirúa } & 8.228 & 0 & 0 & 4.534 & 0 & 12.762 \\
\hline \multicolumn{3}{|c|}{ Total provincia } & 34.597 & 3.160 & 602 & 10.503 & 3.637 & 52.500 \\
\hline
\end{tabular}

L: Ligera

M: Moderada

S: Severa:

SI: Sin Información

SE: Sin erosión

En la provincia de Arauco el $49,3 \%$ de la superficie potencialmente disponible para la forestación se ubica en comunas con IDH calificado como muy bajo, en tanto el $27,2 \%$ está en comunas con IDH bajo.

El 78,2\% de esta superficie potencial provincial corresponde a la Clase de Capacidad de Uso del Suelo VII. Destaca que mayoritariamente estos suelos presentan erosión ligera $(65,9 \%)$ o sin erosión $(20,0 \%)$. ha.

Las comunas de Tirúa y Arauco, presentan superficies disponibles por sobre las 10.000 
Cuadro $\mathrm{N}^{\circ} 4$

SUPERFICIE POTENCIALMENTE DISPONIBLE PARA FORESTACIÓN SEGÚN COMUNA, IDH, CLASES DE CAPACIDAD DE USO DE LOS SUELOS Y CATEGORÍAS DE EROSIÓN. PROVINCIA DE BIOBÍO

\begin{tabular}{|c|c|c|c|c|c|c|c|c|c|}
\hline \multirow{3}{*}{ Comuna } & \multirow{3}{*}{ IDH } & \multirow{3}{*}{$\begin{array}{l}\text { Capacidad } \\
\text { Uso del } \\
\text { Suelo }\end{array}$} & \multicolumn{6}{|c|}{ Cateqorías de Erosión } & \multirow{2}{*}{ Total } \\
\hline & & & $\mathbf{L}$ & $\mathbf{M}$ & $\mathrm{s}$ & MS & SE & SI & \\
\hline & & & \multicolumn{7}{|c|}{ (ha) } \\
\hline \multirow{2}{*}{ Alto Biobío } & \multirow{2}{*}{$\begin{array}{l}\text { Muy } \\
\text { Bajo }\end{array}$} & $\mathrm{Vl}$ & 78 & 0 & 0 & 0 & 0 & 0 & 78 \\
\hline & & VII & 71 & 0 & 0 & 0 & 0 & 0 & 71 \\
\hline \multicolumn{3}{|c|}{ Total Alto Biobío } & 149 & 0 & 0 & 0 & 0 & 0 & 149 \\
\hline \multirow{2}{*}{ Antuco } & \multirow{2}{*}{ Bajo } & $\mathrm{VI}$ & 0 & 358 & 0 & 0 & 227 & 0 & 584 \\
\hline & & VII & 127 & 386 & 0 & 0 & 1.720 & 0 & 2.233 \\
\hline \multicolumn{3}{|c|}{ Total Antuco } & 127 & 744 & 0 & 0 & 1.947 & 0 & 2.818 \\
\hline \multirow{2}{*}{ Cabrero } & \multirow{2}{*}{$\begin{array}{l}\text { Muy } \\
\text { Bajo }\end{array}$} & $\mathrm{VI}$ & 15 & 160 & 0 & 0 & 1.879 & 0 & 2.055 \\
\hline & & VII & 116 & 0 & 742 & 64 & 314 & 772 & 2.008 \\
\hline \multicolumn{3}{|c|}{ Total Cabrero } & 131 & 160 & 742 & 64 & 2.193 & 772 & 4.062 \\
\hline \multirow{2}{*}{ Laja } & \multirow{2}{*}{ Bajo } & $\mathrm{VI}$ & 1.935 & 0 & 0 & 0 & 544 & 0 & 2.478 \\
\hline & & VII & 710 & 964 & 2.083 & 479 & 164 & 38 & 4.438 \\
\hline \multicolumn{3}{|l|}{ Total Laja } & 2.644 & 964 & 2.083 & 479 & 708 & 38 & 6.917 \\
\hline \multirow{2}{*}{$\begin{array}{c}\text { Los } \\
\text { Angeles }\end{array}$} & \multirow{2}{*}{ Medio } & $\mathrm{VI}$ & 161 & 643 & 0 & 0 & 8.034 & 0 & 8.838 \\
\hline & & VII & 529 & 633 & 904 & 0 & 2.216 & 5.620 & 9.902 \\
\hline \multicolumn{3}{|c|}{ Total Los Angeles } & 690 & 1.275 & 904 & 0 & 10.250 & 5.620 & 18.740 \\
\hline Mulabón & Dois & $\mathrm{VI}$ & 5 & 3.931 & 0 & 0 & 10 & 0 & 3.946 \\
\hline Mulchen & Вајо & VII & 15 & 1.019 & 2.730 & 0 & 11 & 34 & 3.809 \\
\hline Total Mulch & & & 20 & 4.950 & 2.730 & 0 & 22 & 34 & 7.756 \\
\hline Alorimiont & Roip & $\mathrm{VI}$ & 937 & 1.543 & & 0 & 426 & 0 & 2.906 \\
\hline Nacımıento & Вајо & VII & 324 & 4.818 & 3.233 & 4.502 & 0 & 81 & 12.958 \\
\hline Total Nacin & & & 1.261 & 6.361 & 3.233 & 4.502 & 426 & 81 & 15.865 \\
\hline Nloaroto & Muy & $\mathrm{VI}$ & 4 & 0 & 0 & 0 & 119 & 0 & 123 \\
\hline Negrete & Bajo & VII & 0 & 60 & 48 & 0 & 0 & 1.736 & 1.844 \\
\hline Total Negre & & & 4 & 60 & 48 & 0 & 119 & 1.736 & 1.967 \\
\hline Puilar & & $\mathrm{VI}$ & 24 & 1.455 & 0 & 0 & 0 & 0 & 1.478 \\
\hline Quilaco & Bajo & VII & 447 & 595 & 143 & 0 & 0 & 0 & 1.185 \\
\hline Total Quila & & & 470 & 2.049 & 143 & 0 & 0 & 0 & 2.663 \\
\hline Quillose & Rain & $\mathrm{VI}$ & 15 & 2.413 & 0 & 0 & 2.425 & 0 & 4.853 \\
\hline Quilleco & Вајо & VII & 124 & 434 & 483 & 0 & 1.426 & 258 & 2.725 \\
\hline Total Quille & & & 138 & 2.847 & 483 & 0 & 3.851 & 258 & 7.578 \\
\hline San & Roin & $\mathrm{VI}$ & 130 & 273 & 0 & 0 & 0 & 0 & 403 \\
\hline Rosendo & Вајо & VII & 42 & 17 & 406 & 1.101 & 0 & 27 & 1.592 \\
\hline Total San P & endo & & 172 & 289 & 406 & 1.101 & 0 & 27 & 1.996 \\
\hline Santa & Muy & $\mathrm{VI}$ & 0 & 1.869 & 0 & 0 & 58 & 0 & 1.928 \\
\hline Bárbara & Bajo & VII & 2 & 1.624 & 211 & 0 & 55 & 0 & 1.893 \\
\hline Total Santa & árbara & & 2 & 3.493 & 211 & 0 & 113 & 0 & 3.820 \\
\hline & & $\mathrm{VI}$ & 1.457 & 518 & 0 & 0 & 481 & 0 & 2.456 \\
\hline Tucapel & Bajo & VII & 185 & 675 & 0 & 0 & 1.135 & 0 & 1.996 \\
\hline Total Tucap & & & 1.642 & 1.193 & 0 & 0 & 1.616 & 0 & 4.452 \\
\hline & Muy & $\mathrm{VI}$ & 4.234 & 1.136 & 0 & 0 & 56 & 0 & 5.426 \\
\hline Yumbel & Bajo & VII & 401 & 4.989 & 6.681 & 7.758 & 539 & 254 & 20.621 \\
\hline Total Yumb & & & 4.634 & 6.125 & 6.681 & 7.758 & 595 & 254 & 26.047 \\
\hline Total & & & 12.087 & 30.511 & 17.666 & 13.904 & 21.839 & 8.821 & 104.828 \\
\hline
\end{tabular}


El $45,2 \%$ de la superficie potencial disponible para la forestación en la provincia del Biobío, se ubica en comunas con IDH bajo, y el $36,9 \%$ presentan IDH muy bajo. Mayoritariamente corresponden a la Clase VII (64,2\%) y en cuanto a erosión en el $29,1 \%$ esta es moderada, en el $20,8 \%$, no la hay y en el $16,8 \%$ es severa. Yumbel es la comuna con mayor superficie disponible a nivel regional y junto con las comunas de Los Angeles y Nacimiento poseen superficies superiores a las 10.000 ha.

\section{Cuadro $N^{\circ} 5$
SUPERFICIE POTENCIALMENTE DISPONIBLE PARA FORESTACIÓN SEGÚN COMUNA, IDH, CLASES DE CAPACIDAD DE USO DE LOS SUELOS Y CATEGORÍAS DE EROSIÓN. PROVINCIA DE CONCEPCIÓN}

\begin{tabular}{|c|c|c|c|c|c|c|c|c|c|}
\hline \multirow{3}{*}{ Comuna } & \multirow{3}{*}{ IDH } & \multirow{3}{*}{$\begin{array}{c}\text { Capacidad } \\
\text { Uso del } \\
\text { Suelo }\end{array}$} & \multicolumn{6}{|c|}{ Categorías de Erosión } & \multirow{2}{*}{ Total } \\
\hline & & & $\mathbf{L}$ & M & $\mathrm{S}$ & MS & SE & SI & \\
\hline & & & \multicolumn{7}{|c|}{ (ha) } \\
\hline Chiguayante & Muy Alto & VII & 0 & 273 & 0 & 0 & 0 & 0 & 273 \\
\hline \multicolumn{3}{|c|}{ Total Chiguayante } & 0 & 273 & 0 & 0 & 0 & 0 & 273 \\
\hline \multirow{2}{*}{ Concepción } & \multirow{2}{*}{ Muy Alto } & $\mathrm{VI}$ & 133 & 0 & 0 & 0 & 87 & 0 & 220 \\
\hline & & VII & 0 & 2.033 & 192 & 0 & 0 & 0 & 2.225 \\
\hline \multicolumn{3}{|c|}{ Total Concepción } & 133 & 2.033 & 192 & 0 & 87 & 0 & 2.445 \\
\hline \multirow{2}{*}{ Coronel } & \multirow{2}{*}{ Alto } & $\mathrm{VI}$ & 98 & 0 & 0 & 0 & & 0 & 98 \\
\hline & & VII & 974 & 0 & 0 & 0 & 73 & 102 & 1.149 \\
\hline \multicolumn{3}{|l|}{ Total Coronel } & 1.072 & 0 & 0 & 0 & 73 & 102 & 1.247 \\
\hline \multirow{2}{*}{ Florida } & \multirow{2}{*}{ Muy Bajo } & $\mathrm{VI}$ & 2.959 & 64 & 0 & 0 & 0 & 0 & 3.024 \\
\hline & & VII & 0 & 12.701 & 2.607 & 1.327 & 0 & 0 & 16.635 \\
\hline \multicolumn{3}{|l|}{ Total Florida } & 2.959 & 12.765 & 2.607 & 1.327 & 0 & 0 & 19.659 \\
\hline \multirow{2}{*}{ Hualpén } & \multirow{2}{*}{ Alto } & $\mathrm{VI}$ & 162 & 0 & 0 & 0 & 0 & 0 & 162 \\
\hline & & VII & 401 & 0 & 0 & 0 & 0 & 0 & 401 \\
\hline \multicolumn{3}{|l|}{ Total Hualpén } & 564 & 0 & 0 & 0 & 0 & 0 & 564 \\
\hline \multirow{2}{*}{ Hualqui } & \multirow{2}{*}{ Bajo } & $\mathrm{VI}$ & 965 & 0 & 0 & 0 & 0 & 0 & 965 \\
\hline & & VII & 343 & 6.022 & 1.459 & 0 & 879 & 0 & 8.703 \\
\hline \multicolumn{3}{|l|}{ Total Hualqui } & 1.308 & 6.022 & 1.459 & 0 & 879 & 0 & 9.667 \\
\hline \multirow{2}{*}{ Lota } & \multirow{2}{*}{ Bajo } & $\mathrm{VI}$ & 0 & 0 & 0 & 0 & 12 & 0 & 12 \\
\hline & & VII & 9 & 0 & 0 & 0 & 0 & 0 & 9 \\
\hline Total Lota & & & 9 & 0 & 0 & 0 & 12 & 0 & 21 \\
\hline \multirow{2}{*}{ Penco } & Medin & $\mathrm{VI}$ & 161 & 0 & 0 & 0 & 56 & 0 & 218 \\
\hline & IVledio & VII & 30 & 295 & 718 & 0 & 0 & 0 & 1.044 \\
\hline Total Penco & & & 192 & 295 & 718 & 0 & 56 & 0 & 1.261 \\
\hline San Pedro & Mux Alto & $\mathrm{VI}$ & 10 & 0 & 0 & 0 & 43 & 0 & 53 \\
\hline de la Paz & IVIuY AilO & VII & 170 & 0 & 0 & 0 & 0 & 110 & 279 \\
\hline Total San Pec & ro de la $\mathrm{Pa}$ & & 180 & 0 & 0 & 0 & 43 & 110 & 332 \\
\hline Santa Juana & Muv Rain & $\mathrm{VI}$ & 1.339 & 2.405 & 0 & 0 & 0 & 0 & 3.744 \\
\hline santa Juana & IVluy вајо & VII & 189 & 4.840 & 1.658 & 4.602 & 33 & 156 & 11.478 \\
\hline Total Santa J & uana & & 1.528 & 7.245 & 1.658 & 4.602 & 33 & 156 & 15.222 \\
\hline Talcahuano & Alto & $\mathrm{VI}$ & 164 & 0 & 0 & 0 & 0 & 0 & 164 \\
\hline & & VII & 637 & 0 & 0 & 0 & 0 & 0 & 637 \\
\hline Total Talcahu & ano & & 801 & 0 & 0 & 0 & 0 & 0 & 801 \\
\hline & & $\mathrm{VI}$ & 1.805 & 0 & 0 & 0 & 0 & 0 & 1.805 \\
\hline Tomé & Medio & VII & 313 & 4.061 & 2.223 & 0 & 0 & 0 & 6.597 \\
\hline Total Tomé & & & 2.119 & 4.061 & 2.223 & 0 & 0 & 0 & 8.402 \\
\hline Total & & & 10.864 & 32.695 & 8.857 & 5.929 & 1.184 & 368 & 59.895 \\
\hline
\end{tabular}


Concepción es la única provincia de la región que presenta las cinco categorías de IDH en sus comunas y en el $58,2 \%$ de la superficie potencial para la forestación este índice se ubica en el nivel muy bajo. Mayoritariamente esta superficie pertenece a la Clase Capacidad de Uso del Suelo VII (82,5\%) y en cuanto a las categorías de erosión, el $54,6 \%$ está clasificado como erosión moderada, el $24,7 \%$ pertenece a severa o muy severa y el $20,1 \%$ a erosión ligera o sin erosión. Las comunas de Florida y Santa Juana presentan superficies potenciales por sobre las 10.000 ha.

\section{SUPERFICIE POTENCIALMENTE DISPONIBLE PARA FORESTACIÓN SEGÚN COMUNA, IDH, CLASES DE CAPACIDAD DE USO DE LOS SUELOS Y CATEGORÍAS DE EROSIÓN. PROVINCIA DE ÑUBLE}

\begin{tabular}{|c|c|c|c|c|c|c|c|c|}
\hline \multirow{3}{*}{ Comuna } & \multirow{3}{*}{ IDH } & \multirow{3}{*}{$\begin{array}{l}\text { Capacidad } \\
\text { Uso del } \\
\text { Suelo }\end{array}$} & \multicolumn{5}{|c|}{ Cateqorías de Erosión } & \multirow{2}{*}{ Total } \\
\hline & & & $\mathbf{L}$ & $\mathbf{M}$ & $\mathrm{S}$ & MS & SE & \\
\hline & & & \multicolumn{6}{|c|}{ (ha) } \\
\hline \multirow{2}{*}{ Bulnes } & \multirow{2}{*}{ Muy Bajo } & $\mathrm{VI}$ & 0 & 76 & 0 & 0 & 1.807 & 1.883 \\
\hline & & VII & 0 & 267 & 218 & 0 & 429 & 914 \\
\hline \multicolumn{3}{|l|}{ Total Bulnes } & 0 & 343 & 218 & 0 & 2.236 & 2.797 \\
\hline \multirow{2}{*}{ Chillán } & \multirow{2}{*}{ Alto } & $\mathrm{VI}$ & 5 & 226 & & 0 & 591 & 821 \\
\hline & & VII & 148 & 406 & 1.719 & 235 & 605 & 3.113 \\
\hline \multicolumn{3}{|l|}{ Total Chillán } & 153 & 632 & 1.719 & 235 & 1.195 & 3.934 \\
\hline \multirow{2}{*}{$\begin{array}{l}\text { Chillán } \\
\text { Viejo }\end{array}$} & \multirow{2}{*}{ Alto } & $\mathrm{VI}$ & 80 & 222 & 0 & 0 & 524 & 826 \\
\hline & & VII & 0 & 1.189 & 611 & 17 & 1.112 & 2.929 \\
\hline \multicolumn{3}{|c|}{ Total Chillán Viejo } & 80 & 1.411 & 611 & 17 & 1.636 & 3.755 \\
\hline \multirow{2}{*}{ Cobquecura } & \multirow{2}{*}{ Muy Bajo } & $\mathrm{VI}$ & 926 & 209 & 0 & 0 & 1.293 & 2.428 \\
\hline & & VII & 1.259 & 4.309 & 371 & 37 & 0 & 5.977 \\
\hline \multicolumn{2}{|c|}{ Total Cobquecura } & & 2.185 & 4.519 & 371 & 37 & 1.293 & 8.405 \\
\hline \multirow{2}{*}{ Coelemu } & \multirow{2}{*}{ Muy Bajo } & $\mathrm{VI}$ & 1.858 & 24 & 0 & 0 & 0 & 1.882 \\
\hline & & VII & & 4.723 & 47 & 0 & 0 & 4.770 \\
\hline \multicolumn{3}{|c|}{ Total Coelemu } & 1.858 & 4.747 & 47 & 0 & 0 & 6.652 \\
\hline & \multirow{2}{*}{ Muy Bajo } & $\mathrm{VI}$ & 100 & 554 & 0 & 0 & 27 & 680 \\
\hline & & VII & 8 & 896 & 0 & 0 & 0 & 904 \\
\hline \multicolumn{3}{|c|}{ Total Coihueco } & 108 & 1.450 & 0 & 0 & 27 & 1.585 \\
\hline \multirow{2}{*}{ El Carmen } & Mum Poir & $\mathrm{VI}$ & 6.103 & 1.054 & 0 & 0 & 0 & 7.157 \\
\hline & Muy Вајo & VII & 35 & 2.446 & 0 & 0 & 215 & 2.696 \\
\hline Total El Car & & & 6.139 & 3.499 & 0 & 0 & 215 & 9.853 \\
\hline & & $\mathrm{VI}$ & 156 & 2.687 & 0 & 0 & 197 & 3.041 \\
\hline Ninhue & Muy Вајо & VII & 1.166 & 0 & 8.461 & 95 & 0 & 9.722 \\
\hline Total Ninhue & & & 1.322 & 2.687 & 8.461 & 95 & 197 & 12.763 \\
\hline Ñiauén & Muv Baio & $\mathrm{VI}$ & 3 & 43 & 0 & 0 & 1.028 & 1.074 \\
\hline Niquen & Muy Bajo & VII & 41 & 19 & 0 & 0 & 0 & 60 \\
\hline Total Ñiquér & & & 44 & 62 & 0 & 0 & 1.028 & 1.134 \\
\hline & & $\mathrm{VI}$ & 2.201 & 0 & 0 & 0 & 10 & 2.211 \\
\hline Pemuco & Muy Bajo & VII & 0 & 238 & 133 & 0 & 44 & 415 \\
\hline Total Pemuc & & & 2.201 & 238 & 133 & 0 & 54 & 2.626 \\
\hline & & $\mathrm{VI}$ & 1.913 & 311 & 0 & 0 & 0 & 2.225 \\
\hline Pinto & Muy Вајо & VII & 206 & 924 & 0 & 0 & 69 & 1.199 \\
\hline Total Pinto & & & 2.119 & 1.235 & 0 & 0 & 69 & 3.423 \\
\hline & & $\mathrm{VI}$ & 379 & 660 & 0 & 0 & 0 & 1.039 \\
\hline Portezuelo & Muy Вајо & VII & 0 & 0 & 8.093 & 34 & 0 & 8.127 \\
\hline Total Portez & & & 379 & 660 & 8.093 & 34 & 0 & 9.166 \\
\hline
\end{tabular}


Cuadro $N^{\circ} 6$. Continuación

\begin{tabular}{|c|c|c|c|c|c|c|c|c|}
\hline \multirow{2}{*}{ Quillón } & \multirow{2}{*}{ Muy Bajo } & $\mathrm{VI}$ & 0 & 1.120 & 0 & 0 & 164 & 1.284 \\
\hline & & VII & 275 & 1.813 & 5.699 & 5.743 & 54 & 13.583 \\
\hline \multicolumn{3}{|l|}{ Total Quillón } & 275 & 2.932 & 5.699 & 5.743 & 218 & 14.867 \\
\hline \multirow{2}{*}{ Quirihue } & \multirow{2}{*}{ Muy Bajo } & $\mathrm{VI}$ & 415 & 1.722 & 0 & 0 & 1.162 & 3.299 \\
\hline & & VII & 4.002 & 2.138 & 3.513 & 1.678 & 0 & 11.331 \\
\hline \multicolumn{3}{|c|}{ Total Quirihue } & 4.416 & 3.860 & 3.513 & 1.678 & 1.162 & 14.629 \\
\hline \multirow{2}{*}{ Ranquil } & \multirow{2}{*}{ Muy Bajo } & $\mathrm{VI}$ & 707 & 734 & 0 & 0 & 0 & 1.441 \\
\hline & & VII & 0 & 2.331 & 2.161 & 563 & 127 & 5.182 \\
\hline \multicolumn{3}{|l|}{ Total Ranquil } & 707 & 3.064 & 2.161 & 563 & 127 & 6.622 \\
\hline \multirow{2}{*}{ San Carlos } & \multirow{2}{*}{ Bajo } & $\mathrm{VI}$ & 13 & 1.394 & 0 & 0 & 1.090 & 2.497 \\
\hline & & VII & 1.443 & 503 & 2.096 & 408 & 0 & 4.450 \\
\hline \multicolumn{3}{|c|}{ Total San Carlos } & 1.456 & 1.897 & 2.096 & 408 & 1.090 & 6.947 \\
\hline \multirow{2}{*}{ San Fabián } & \multirow{2}{*}{ Muy Bajo } & $\mathrm{VI}$ & 20 & 370 & 0 & 0 & 0 & 390 \\
\hline & & VII & 0 & 12 & 0 & 0 & 0 & 12 \\
\hline \multicolumn{3}{|c|}{ Total San Fabián } & 20 & 382 & 0 & 0 & 0 & 402 \\
\hline \multirow{2}{*}{ San Ignacio } & \multirow{2}{*}{ Muy Bajo } & $\mathrm{VI}$ & 1.849 & 0 & 0 & 0 & 176 & 2.025 \\
\hline & & VII & & 338 & 13 & 0 & 464 & 815 \\
\hline \multicolumn{3}{|c|}{ Total San Ignacio } & 1.849 & 338 & 13 & 0 & 640 & 2.840 \\
\hline \multirow{2}{*}{ San Nicolás } & \multirow{2}{*}{ Muy Bajo } & $\mathrm{VI}$ & 429 & 714 & 0 & 0 & 1.034 & 2.177 \\
\hline & & VII & 275 & 162 & 5.086 & 25 & & 5.548 \\
\hline \multicolumn{3}{|c|}{ Total San Nicolás } & 704 & 876 & 5.086 & 25 & 1.034 & 7.725 \\
\hline \multirow{2}{*}{ Treguaco } & \multirow{2}{*}{ Muy Bajo } & $\mathrm{VI}$ & 46 & 805 & 0 & 0 & 178 & 1.029 \\
\hline & & VII & 32 & 582 & 4.462 & 3.010 & 0 & 8.085 \\
\hline \multicolumn{3}{|c|}{ Total Treguaco } & 77 & 1.387 & 4.462 & 3.010 & 178 & 9.114 \\
\hline \multirow{2}{*}{ Yungay } & \multirow{2}{*}{ Medio } & $\mathrm{VI}$ & 2.656 & 23 & 0 & 0 & 1.054 & 3.733 \\
\hline & & VII & 86 & 404 & 31 & 0 & 820 & 1.341 \\
\hline \multicolumn{3}{|l|}{ Total Yungay } & 2.742 & 427 & 31 & 0 & 1.874 & 5.074 \\
\hline \multicolumn{3}{|l|}{ Total } & 28.835 & 36.648 & 42.714 & 11.844 & 14.273 & 134.314 \\
\hline
\end{tabular}

La provincia de Ñuble en tanto presenta la mayor superficie potencial para la forestación en la región y el $85,3 \%$ de esta pertenece a comunas con IDH calificado como muy bajo. Las comunas de Quillón, Quirihue y Ninhue presentan superficies superiores a las 10.000 ha.

El $67,9 \%$ de la superficie potencial provincial, corresponde a la Clase de Capacidad de Uso del Suelo VII. La categoría erosión severa representa el $31,8 \%$ de la superficie provincial potencial; seguida de suelos con erosión moderada $(27,3 \%)$ y erosión ligera $(21,5 \%)$.

En la región del Biobío existen diez comunas con superficies potencialmente disponibles mayores a 10.000 ha, las que acumulan 162.874 ha, representando el 46,3\% de la superficie potencial regional.

\section{DISCUSIÓN Y CONCLUSIONES}

La región del Biobío tiene una superficie total de 3,71 millones de hectáreas, y el 43,84\% de esta superficie corresponde a suelos de las Clases de Capacidad de Uso VI y VII (1,62 millones de hectáreas), muy mayoritariamente Clase VII (1,29 millones de hectáreas), lo que confirma que esta es una región con fuerte vocación forestal.

De la superficie de suelos forestales, 351.540 ha $(21,6 \%)$ son suelos potencialmente disponibles para forestación, superficie que se obtiene al descontar las cubiertas actuales de bosque nativo y plantaciones forestales, además de variantes como escoriales de lava, cursos de 
agua y otros que se extraen del análisis. La superficie obtenida en el estudio ha sido definida como potencialmente disponible, dado que en la práctica esta disponibilidad está condicionada por múltiples otros factores no incluidos en esta primera aproximación.

La región del Biobío concentra la mayor parte de la industria forestal del país y el $38 \%$ de las plantaciones forestales nacionales. La superficie de suelos potencialmente disponible para la forestación corresponde al 9,5\% de la superficie regional (3,71 millones de hectáreas), al 24\% de los suelos forestales (bosque nativo y plantaciones forestales), y equivale al $38,2 \%$ de la superficie regional actual de plantaciones (0,92 millones de hectáreas).

La superficie de suelos potencialmente disponible para forestación está compuesta en un $70,8 \%$ por suelos clase VII (248.982 ha) y el $29,1 \%$ restante por suelos clase VI.

En los suelos clases VI y VII potencialmente disponibles para forestación, el 28,9\% de la superficie presenta erosión severa a muy severa (101.516 ha), situación que afecta principalmente a aquellos de clase VII, los que debiesen ser objeto de políticas que fomenten su recuperación.

De acuerdo a la división administrativa de la región según provincias, la superficie de suelos potencialmente disponible para forestación se concentra en las dos de mayor tamaño, $38,2 \%$ (134.314 ha) en la provincia de Ñuble, un 29,8\% (104.828 ha) en la provincia de Biobío; les siguen con un $17,1 \%$ (59.895 ha) la provincia de Concepción y con un $14,9 \%$ (52.500 ha) la provincia de Arauco.

En materia de erosión de suelos, resaltan las provincias de Nuble y Biobío en las cuales sobre un tercio de la superficie potencialmente disponible para forestación, está dada por suelos con erosión severa a muy severa. Contrariamente, la provincia de Arauco presenta la menor degradación de suelos, con el $1 \%$ de aquellos potencialmente disponibles para forestación en la categoría erosión severa.

Al aplicar la división administrativa según comunas se encuentra que en todas ellas (54) existe superficie potencialmente disponible para forestación y destacan 10 comunas en que esta es superior a 10.000 ha; 2 en la provincia de Arauco, 3 en la provincia de Biobío, 2 en la provincia de Concepción y 3 en la provincia de Ñuble, y estas en conjunto reúnen el $46 \%$ de la superficie total potencialmente disponible para forestación en la región.

Al analizar la superficie potencialmente disponible con el Índice de Desarrollo Humano (IDH), se aprecia que esta se distribuye principalmente en comunas con IDH muy bajo 214.089 ha $(60,9 \%)$ y bajo $(22,3 \%)$.

La provincia de Ñuble acumula 114.604 ha de la superficie potencial en comunas del quintil con menor IDH. De las diez comunas con superficie potencial disponible por sobre las 10 mil hectáreas, siete de ellas (Yumbel, Florida, Santa Juana, Quillón, Quirihue, Ninhue y Tirúa) presentan IDH muy bajo. Estos antecedentes debiesen orientar tanto políticas públicas, como acciones de privados, para focalizar los recursos en comunidades locales y consecuentemente mejorar el desarrollo regional.

Dada la escala de trabajo y la información con que se alimentó el SIG, con distintos grados de resolución espacial y fechas de elaboración, los resultados tienen validez para grandes áreas, a nivel comunal. Análisis más detallados, para zonas específicas, requieren necesariamente de información más precisa.

Desde el punto de vista silvícola, sería de gran interés ahondar el análisis realizado incorporando variables de clima, como precipitación media anual (isoyetas), duración del período seco y temperaturas mínimas absolutas (isotermas), con el fin de delimitar áreas en donde habría que emplear especies forestales distintas a las habitualmente empleadas en la forestación. 
Una cantidad de otras variables deben ser incorporadas para una mejor definición de las superficies disponibles para forestación, relacionadas con topografía y accesibilidad, distancias a centros industriales o de acopio y otras muy importantes relacionadas con la propiedad y el dominio y el uso actual del suelo. Las primeras muy probablemente reducirían las superficies disponibles en la práctica en las zonas orientales de las provincias de Nuble y Biobío, y las segundas son las que podrían explicar las superficies disponibles hacia las zonas costeras de las provincias de Arauco, Concepción y Ñuble.

En este último caso, suelos clase VI cuyo uso actual es ganadería, viñedos u otros se descontarían de la superficie disponible para forestación, igual cosa ocurriría con áreas con atomización de la propiedad que podría ser la situación en algunas comunas de la costa como Tomé, Lebu y otras, y evidentemente con áreas en donde hay conflictos de propiedad como es el caso principalmente de comunas del sur de la provincia de Arauco.

La incorporación de las variables mencionadas y otras permitiría afinar la información en materia de superficies disponible para forestación y especies a emplear, y probablemente sería un valiosa apoyo en la aplicación de políticas públicas orientadas al fomento estatal para la forestación, considerando la priorización de áreas a cubrir y los principales objetivos de la forestación, que podrían estar enfocados en aspectos productivos, en la generación de empleos, en la conservación de suelos, en la regulación de escorrentías, en la producción de biomasa para energía, en la captura de carbono u otros fines, tal vez con montos diferenciados según comunas y objetivos de la forestación.

No obstante lo anterior, los resultados de este estudio, sobre superficies potencialmente disponibles para forestación en la región, se espera sean antecedentes orientadores para la gestión de las autoridades regionales, para las empresas forestales de la región y para pequeños y medianos inversionistas interesados en el establecimiento de plantaciones forestales.

\section{REFERENCIAS}

CIREN, 2006. Zonificación de Erosión y Fragilidad de los Suelos del Secano Costero de las Regiones VI y VII. Boletín Técnico de Resultados. Santiago, Chile. 65p.

CONAF, CONAMA, BIRF, 1999: Catastro y Evaluación de Recursos Vegetacionales Nativos de Chile. Proyecto CONAF-CONAMA-BIRF.

CORMA, 2014. Aportes a la Economía. Empleo.

[En línea] http://www.corma.cl/perfil-del-sector/aportes-a-la-economia/empleo [consulta: 14 julio 2014].

Flores, Juan; Martínez, Eduardo; Espinosa, Marión; Henríquez, Gabriel; Avendaño, Pablo; Torres, Patricio y Ahumada, Isaac, 2010. Determinación de la erosión actual y potencial de los suelos de Chile; Región del Bío Bío. Publicación N¹48 CIREN. Santiago, Chile. 47p.

INFOR, 2013. Anuario Forestal 2013. Boletín estadístico №140. Instituto Forestal. Santiago, Chile.148 p.

INFOR, 2014. Estadísticas Forestales. Instituto Forestal.

[En línea] http://wef.infor.cl/consultas_linea/consultaenlinea.php [consulta: 28 junio 2014].

Lignum, 2014. Concentración del Cluster Forestal. Revista Julio año 24 №150. pp.34-37.

ODEPA, 2013. ICET. Sistema de Consulta Estadístico Territorial, Cartografía Digital. Oficina de Estudios y Políticas Agrarias ODEPA. [en línea] http://icet.odepa.cl/

PNUD - MIDEPLAN. 2005. Las trayectorias del desarrollo humano en las comunas de Chile (1994-2003). №11 Temas de desarrollo sustentable. Santiago, Chile. 150p. 